\title{
Analysis of SSR information in the pistil transcriptome and the development and characterization of novel tri-nucleotide SSR
} markers in Corylus

\author{
Tiantian Zhao, Jingjing Li, Dingding Miao, Lisong Liang, Guixi Wang and Qinghua Ma \\ Research Institute of Forestry, Chinese Academy of Forestry, Key Laboratory of Tree Breeding and Cultivation of the State \\ Forestry and Grassland Administration, Hazelnut Engineering and Technical Research Center of the State Forestry and \\ Grassland Administration, Beijing, China
}

\begin{abstract}
Summary
Hazelnuts (Corylus) are one of the most important nut crops and woody oil plants, having high economic and nutritional value. It is important to study the taxonomy, phylogenetics, and genetic diversity in the genus Corylus. In this study, we used the previously constructed pistil transcriptome of the Ping'ou hybrid hazelnut cultivar 'Dawei' to analyze the SSR information and develop novel tri-nucleotide SSR markers in Corylus. The results identified 16,339 sequences containing SSRs, resulting in a percentage of $19.72 \%$. An SSR locus was identified for every $5.67 \mathrm{~kb}$ of the examined sequences, and 497 motif sequence types were identified. The di-nucleotide repeat type $(63.82 \%)$ was the most abundant, followed by the tri-nucleotide repeat type $(24.54 \%)$. (AAG/CTT)n repeats were dominant among the tri-nucleotide repeats, followed by (AAT/ATT)n, (ATC/ATG)n, (AGG/CCT)n and (ACC/ GGT)n repeats. The sequences with a tri-nucleotide repeat were used to design 160 primer pairs, of which 122 primer pairs showed successful PCR amplification in 'Dawei'. Twenty-two primer pairs that showed polymorphism among the 13 tested samples of the three Corylus species were selected, with an average PIC value of 0.50. A dendrogram based on 82 alleles of the 13 samples using the developed SSR primer pairs was generated. The samples were clustered into three main groups in accordance with the species and showed a complete separation in the dendrogram. In conclusion, transcriptome sequencing is an effective tool for SSR marker development, and the newly developed SSR primer pairs will help in genetic and breeding studies in Corylus and related species.
\end{abstract}

\section{Keywords}

hazelnut, molecular markers, polymorphism, SSR distribution, tri-nucleotide repeats

\section{Introduction}

Hazelnuts (Corylus) are one of the most important nut crops and woody oil plants, and have a very high economic and nutritional value. The genus Corylus is widely distributed across temperate regions of the northern hemisphere. There have been different opinions regarding the taxonomy of Corylus, which is most commonly considered a member of the Birch family, Betulaceae and of the order Fagales

\section{Significance of this study}

What is already known on this subject?

- Hazelnuts (Corylus) are one of the most important nut crops having high value. It is important to study the taxonomy, phylogenetics and genetic diversity in the genus Corylus. Tri-nucleotide repeats were found to be more robust than di-nucleotide repeats when using capillary electrophoresis.

What are the new findings?

- In this study, we used the pistil transcriptome of the Ping' ou hybrid hazelnut cultivar 'Dawei' to analyze the SSR information. SSR locus was identified for every $5.67 \mathrm{~kb}$ of the examined sequences in the whole transcriptome database. The di-nucleotide repeat type was the most abundant, followed by the tri-nucleotide repeat type. Twenty-two novel tri-nucleotide SSR markers with high PIC value among three species of Corylus were developed.

What is the expected impact on horticulture?

- The results indicating that transcriptome sequencing is an effective tool for the development of SSR markers. The newly developed 22 SSR markers will help in genetic and breeding studies in Corylus and related species.

(Mehlenbacher, 2003; Bassil et al., 2005a; Muehlbauer et al., 2014). However, some taxonomists classify it in the subfamily Corylaceae of the family Betulaceae (Chen et al., 1999; Yoo and Wen, 2002; Bassil et al., 2013). The number of species recognized by the taxonomists ranged from 7 to 25 , depending on the authority (Mehlenbacher, 2003; Boccacci et al., 2015). Among these, the European hazelnut, C. avellana L., is the species of commercial cultivation. In China, there are commonly considered eight hazelnut species and two varieties distributed from $24^{\circ} 31^{\prime} \mathrm{N}$ to $51^{\circ} 42^{\prime} \mathrm{N}$ and $85^{\circ} 55^{\prime} \mathrm{E}$ to $132^{\circ} 12^{\prime} \mathrm{E}$. Taxonomists in China also have different opinions in the division of species and varieties native to China, especially the taxonomic status of $C$. kweichowensis Hu or C. heterophylla Fisch. var. sutchuenensis Franch (Ma et al., 2014). In addition, Ping'ou hybrid hazelnuts (C. heterophylla Fisch. $\times$ C. avellana L.), which are hybrids obtained from Ping hazelnuts from northeast China and European hazelnuts introduced from Italy, have been widely cultivated in twenty-odd provinces of China since 2000 (Liang et al., 2012). Dozens of cultivars and varieties of Ping'ou hybrid hazelnuts are grown 
in China, but only sixteen are the main cultivars. The mix of the cultivars/varieties is one of the primary problems in hazelnut production in China. Thus, it is important to study the taxonomy, phylogenetics and genetic diversity in the genus Corylus.

Microsatellites or simple sequence repeats (SSRs) are tandem repeats of short DNA sequences extensively distributed throughout the whole genome in both coding and non-coding regions and are considered ideal molecular markers. The distinguishing features of SSR loci are multi-allelic, co-dominant inheritance patterns, reproducibility, high polymorphism, locus specificity, and transferability to related species and genera (Powell et al., 1996). SSRs can be derived from genomic sequences (genomic SSRs) and expressed sequence tags (EST-SSRs). Compared with genomic SSRs, EST-SSRs have several special advantages due to either a relatively higher transferability or a potential gene function (Wei et al., 2011), which have been evaluated in many studies (Poncet et al., 2006; Luro et al., 2008; Wang et al., 2010). At present, more than 230 SSR loci have been developed in Corylus (Bassil et al., 2005a, b, 2013; Boccacci et al., 2005; Gürcan and Mehlenbacher, 2010a, b; Gűrcan et al., 2010a), which have been widely used in germplasm identification, genetic diversity, linkage map construction and disease-resistant seedling selection (Mehlenbacher et al., 2006; Boccacci et al., 2006, 2013; Gưrcan et al., 2010b; Sathuvalli et al., 2011; Bassil et al., 2013; Beltramo et al., 2014; Zong et al., 2015). Most of the above markers were developed from microsatellite-enriched libraries, which are derived from genomic sequences. Only 20 EST-SSRs, characterized in Corylus, were derived from an initial whole-transcriptome assembly (Boccacci et al., 2015). The repeat motifs are mainly di-nucleotide repeats, tri-nucleotide repeats and quad-nucleotide repeats possessing a very low ratio. Research showed that di-nucleotide repeats have the disadvantage of high amounts of stuttering, which easily lead to mis-calling, whereas tri-nucleotide repeats and other longer core motifs can significantly reduce arbitrary bindings. Tri-nucleotide repeats were found to be more robust than di-nucleotide repeats when using capillary electrophoresis (Zane et al., 2002; Morgante et al., 2002; Amos et al., 2007; Cipriani et al., 2008; Li et al., 2014).

With the development of next-generation sequencing techniques, transcriptome sequencing offers greater expressed sequence tags, which provide a time- and cost-effective way to develop EST-SSRs, especially for the no-reference genomic species (Mardis, 2008; Morozova and Marra, 2008; Metzker, 2010; Wang et al., 2010; Shi et al., 2011). In our previous study, we performed a pistil transcriptome sequencing during different pollination combinations and stages of Ping'ou hybrid hazelnut cultivars 'Dawei' using Illumina Hiseq 2000. In this study, we analyzed the SSR information of the pistil transcriptome sequencing results and successfully developed 22 tri-nucleotide repeat EST-SSR markers from the sequences with transferability in both $C$. heterophylla and C. avellana. This study developed conserved orthologous tri-nucleotide repeat EST-SSR markers for hazelnuts, which will facilitate gene mapping, linkage map development, genetic diversity study and marker-assisted selective breeding for the genus Corylus.

\section{Materials and methods}

\section{Previous pistil transcriptome sequencing}

In our previous study, we performed 7 groups of transcriptome sequencing during different pollination combina-
TABLE 1. Pollination treatment of the pistils of 'Dawei'.

\begin{tabular}{ll}
\hline Code & Treatment \\
\hline CK & Un-pollinated pistils \\
C0 & Compatible pollination, $0 \mathrm{~h}$ \\
C1 & Compatible pollination, $1 \mathrm{~h}$ \\
C4 & Compatible pollination, $4 \mathrm{~h}$ \\
IC0 & Incompatible pollination, $0 \mathrm{~h}$ \\
IC1 & Incompatible pollination, $1 \mathrm{~h}$ \\
IC4 & Incompatible pollination, $4 \mathrm{~h}$ \\
\hline
\end{tabular}

tions and stages of the pistils of the Ping'ou hybrid hazelnut cultivar 'Dawei' (Table 1). All the pistils used in the 6 treatments were in the full blooming stage. We used the pollen of Ping'ou hybrid hazelnut cultivar 'Liaozhen No. 7' to conduct a compatible pollination, and the pollen of 'Dawei' to conduct an incompatible pollination, based on pollination tests both in the laboratory and in the field. The un-pollinated blooming pistils were used as a control. RNA extraction, library construction, sequencing, De novo assembly and sequence annotation were performed by Beijing Genomics Institute (BGI) (Shenzhen, China). Using the Illumina HiSeq ${ }^{\mathrm{TM}} 2000$ platform, the total sequence output was $42,046,035,660 \mathrm{nt}$; 82,847 Unigenes were assembled and annotated with the total length of 108,232,640 bp (data unpublished).

\section{SSR loci identification and primer design}

The MISA program, MIcroSAtellite (http://pgrc. ipk-gatersleben.de/misa/misa.html) was used to identify the SSR loci. The minimum repeat number was set to six for the dinucleotides, five for the tri- and tetra-nucleotides, and four for the penta- and hexa-nucleotides. Primer premier 5.0 (Premier Biosoft International, CA, USA) was used to design the primer pairs flanking the SSR loci with perfect tri-nucleotide motifs using the following parameters: (1) the primers were 12-25 nucleotides long, and the length of the products ranged from 100 to $300 \mathrm{bp}$; (2) the GC content ranged from $40 \%$ to $70 \%$; (3) the $\mathrm{Tm}$ value ranged from $55^{\circ} \mathrm{C}$ to $65^{\circ} \mathrm{C}$, and the $\mathrm{Tm}$ value of the forward and the reverse primer was no more than $5^{\circ} \mathrm{C}$; (4) the primers were devoid of secondary structure or consecutive tracts of a single nucleotide; and (5) the primer designed area was limited to the middle region, with $30 \mathrm{bp}$ removed from the ends of the sequence. In total, 160 pairs of tri-nucleotide repeat SSR primers were randomly selected to perform the following experiment. The primers were synthesized by the Beijing Genomics Institute (BGI, Shenzhen, China).

\section{PCR amplification and fragment analysis}

The designed primers were first tested for amplification using Hy1, Ping' ou hybrid hazelnut main cultivar 'Dawei' and for polymorphism and transferability using 12 other samples (Table 2). All samples were obtained from our germplasm repository located in Yanqing, Beijing, China. Samples from Hy2 to Hy5 were the other main cultivars of Ping'ou hybrid hazelnuts. Samples from H1 to H4 were wild Ping hazelnuts collected from Zhalantun, the Inner Mongolia Autonomous Region, Dunhua, Jilin Province, Tieling, Liaoning Province and Weichang, Hebei Province, respectively. Samples from A1 to A4 were European hazelnut cultivars collected from the National Clonal Germplasm Repository (NCGR) in Corvallis, Oregon, USA.

DNA was extracted from $0.2 \mathrm{~g}$ of healthy young leaf tissue by using a modified procedure described by Zong et al. 


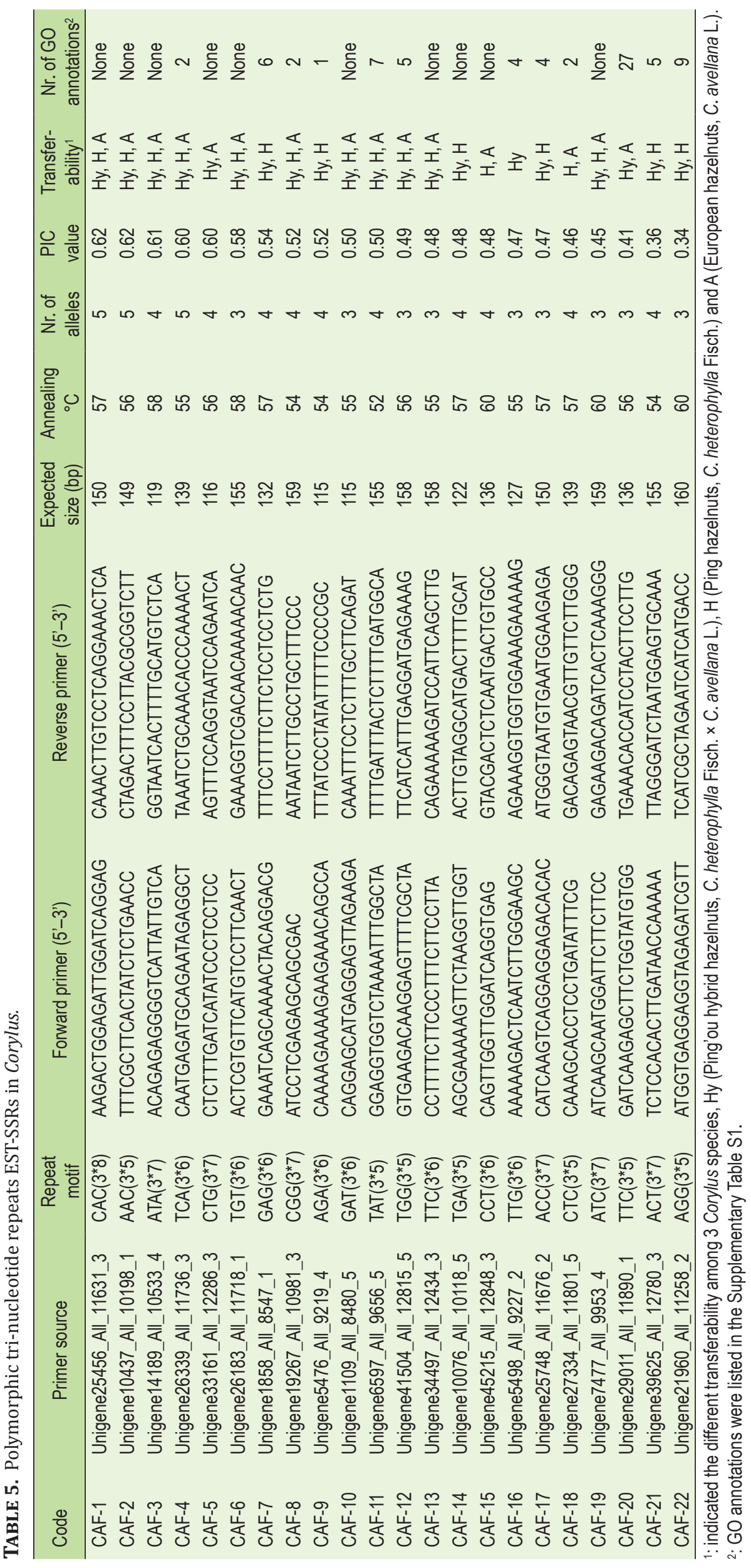


TABLE 2. Plant samples for selected primers.

\begin{tabular}{lll}
\hline Code & Sample name & Latin name \\
\hline Hy1 & Dawei & C. heterophylla Fisch. x \\
Hy2 & Yuzhui & C. avellana L. \\
Hy3 & Liaozhen No. 3 & \\
Hy4 & Liaozhen No. 7 & \\
Hy5 & Liaozhen No. 8 & C. heterophylla Fisch. \\
\hline H1 & Ping-ZLT & \\
H2 & Ping-DH & \\
H3 & Ping-TL & \\
H4 & Ping-WC & C. avellana L. \\
\hline A1 & Willamette & \\
A2 & Hall's Giant & \\
A3 & Tonda Gentile delle Langhe & \\
A4 & Tombul & \\
\hline
\end{tabular}

(2015). The PCR was performed in a total volume of $20.0 \mu \mathrm{L}$ containing $10.0 \mu \mathrm{L} 2 \times$ Taq PCR Mastermix (Tiangen Biotech (Beijing) Co., Ltd.), $0.5 \mathrm{mM}$ each primer, and $10 \mathrm{ng}$ genomic DNA. The PCR conditions were as follows: a pre-denaturation step at $94^{\circ} \mathrm{C}$ for $5 \mathrm{~min}$, then 30 cycles of denaturation $30 \mathrm{~s}$ at $94^{\circ} \mathrm{C}$, annealing $30 \mathrm{~s}$ at the optimal temperature for each primer pair, as reported in Table 5 , and extension $30 \mathrm{~s}$ at $72^{\circ} \mathrm{C}$; the final elongation step was at $72^{\circ} \mathrm{C}$ for $5 \mathrm{~min}$. The PCR products were separated on $6 \%$ denaturing polyacrylamide gels and visualized by silver nitrate staining following the protocol described by Pang et al. (2003).

\section{Data analysis and marker assessment}

EmEditor and R software were used to analyze SSR information in the transcriptome data. The distribution of different repeat types, number and frequency of EST-SSRs were calculated. The frequency of the SSRs was computed as $\mathrm{fc}(\%)=$ $\mathrm{c} / \mathrm{n} \times 100$, where $\mathrm{c}$ is the number of the SSRs identified in the transcriptome data, and $\mathrm{n}$ is the total number of the unigenes assembled and annotated in the transcriptome data.

Bands in the denaturing polyacrylamide gels electrophoresis were scored as their presence (1) or absence (0). Then, the 0,1 matrix was converted into the genotype format. The diversity level of gene loci was evaluated using the polymorphic information content (PIC). The PIC value was calculated according to the formula:

$$
\mathrm{PIC}_{\mathrm{i}}=1-\sum \mathrm{P}_{\mathrm{ij}}^{2}
$$

where $\mathrm{P}_{\mathrm{ij}}$ is the frequency of the $j^{\text {th }}$ allele for the $i^{\text {th }}$ marker (Smith et al., 1997) using PopGen 32 (Version 1.32) and PICCalc (Version 0.6) software. Cluster analysis was performed with an UPGMA (Unweighted Pair Group Method with Arithmetic mean) algorithm based on an SM (Simple Matching) similarity matrix converted from the SSR polymorphism data of 13 tested samples. A dendrogram was generated by using the NTSYS-pc software package (Version 2.10e).

\section{Results}

\section{Identification of SSR loci in the pistil transcriptome of Corylus}

Using the MISA analysis, 16,339 sequences were identified as containing SSRs from 82,847 unigenes, resulting in a percentage of $19.72 \%$. Overall, 19,097 SSRs were identified in 16,339 sequences. The number of sequences containing more than one SSR locus was 2,277, and the number of SSRs present in compound formation was 1,001. An SSR locus was identified for every $5.67 \mathrm{~kb}$ of the examined sequences.

The number of SSRs for di-, tri-, tetra-, penta- and hexa-nucleotide repeat types was 12,188, 4,686, 293, 501 and 428 , respectively. A total of 497 motif sequence types

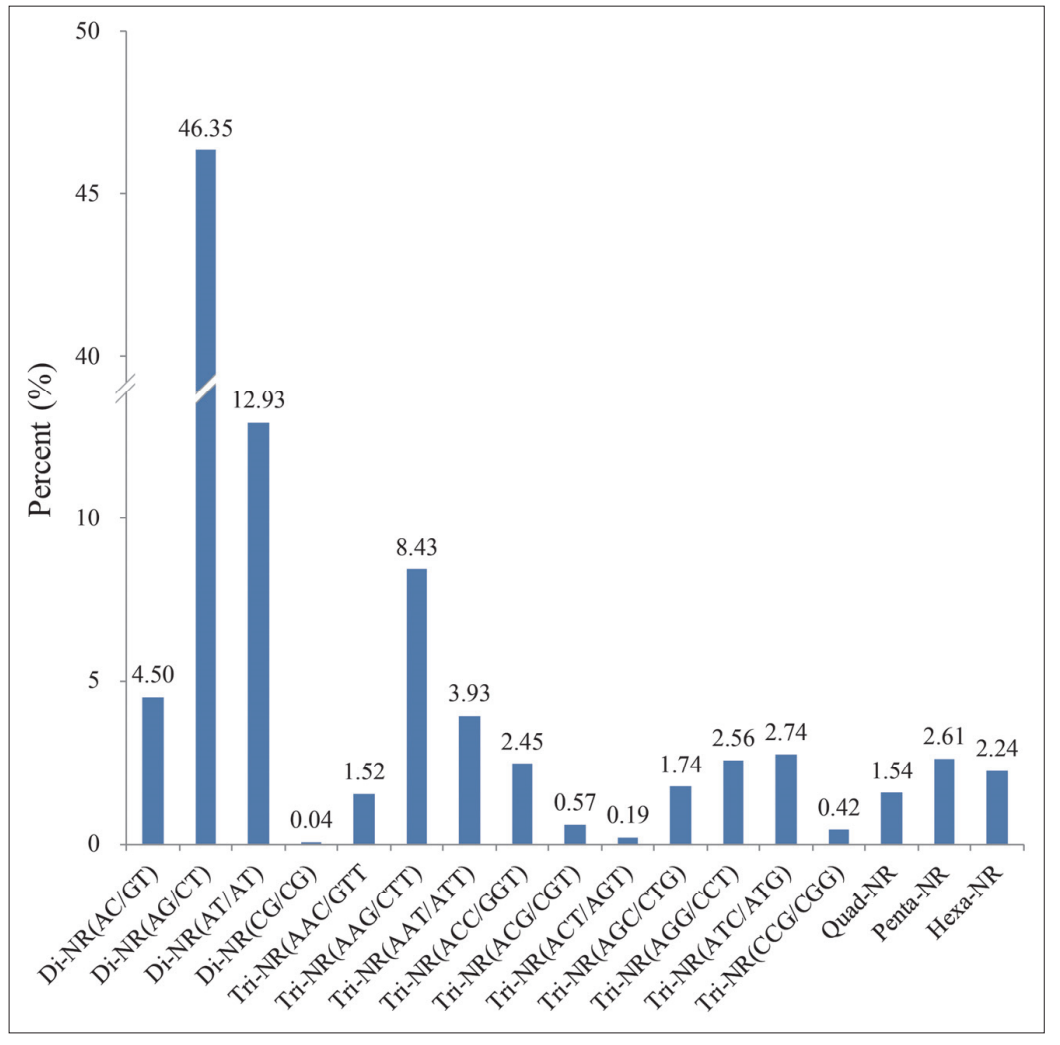

FIGURE 1. Frequency of identified SSR repeat types. 
TABLE 3. Distribution of different repeat types in the pistil transcriptome of Corylus.

\begin{tabular}{lccc}
\hline Unit size & Number of SSRs & Percent (\%) & Frequency (\%) \\
\hline Di-NR & 12,188 & 63.82 & 14.71 \\
Tri-NR & 4,686 & 24.54 & 5.66 \\
Quad-NR & 293 & 1.53 & 0.35 \\
Penta-NR & 501 & 2.62 & 0.60 \\
Hexa-NR & 428 & 2.24 & 0.52 \\
Compound & 1,001 & 5.24 & 1.21 \\
\hline Total & 19,097 & 100 & 23.05 \\
\hline
\end{tabular}

NR: nucleotide repeats.

TABLE 4. Type, number and percentage of SSRs in Corylus.

\begin{tabular}{|c|c|c|c|c|c|c|c|c|c|}
\hline \multirow{2}{*}{ Repeat type } & \multicolumn{9}{|c|}{ Number of repeats } \\
\hline & 4 & 5 & 6 & 7 & 8 & 9 & 10 & 11 & 12 \\
\hline Di-NR & 0 & 0 & 3,200 & 2,249 & 2,326 & 2,224 & 1,673 & 498 & 18 \\
\hline Tri-NR & 0 & 2,342 & 1,422 & 790 & 129 & 0 & 2 & 0 & 1 \\
\hline Quad-NR & 0 & 236 & 57 & 0 & 0 & 0 & 0 & 0 & 0 \\
\hline Penta-NR & 465 & 36 & 0 & 0 & 0 & 0 & 0 & 0 & 0 \\
\hline Hexa-NR & 427 & 1 & 0 & 0 & 0 & 0 & 0 & 0 & 0 \\
\hline Total & 892 & 2,615 & 4,679 & 3,039 & 2,455 & 2,224 & 1,675 & 498 & 19 \\
\hline Percentage & 4.67 & 13.69 & 24.50 & 15.91 & 12.86 & 11.65 & 8.77 & 2.61 & 0.10 \\
\hline
\end{tabular}

NR: nucleotide repeats.

were identified, including 12, 62, 69, 119 and 237 of the di-, tri-, tetra-, penta- and hexa-nucleotide repeat types, respectively (Figure 1 and Tables 3 and 4). Among the perfect SSRs, the di-nucleotide repeat type $(63.82 \%)$ was the most abundant, followed by the tri-nucleotide repeat type (24.54\%). The frequency of the di- and tri-nucleotide repeat types in all the assembled unigenes was $14.71 \%$ and $5.66 \%$, respectively. The tetra-, penta- and hexa-nucleotide motifs were comparatively less frequent, and together comprised only $6.39 \%$ of the total identified SSRs (Table 3). The repeat times of different SSR motifs were distributed from 4 to 12, among which 5 to 9 repeats were the most abundant, with a percentage of $78.61 \%$ among the identified SSRs. For Corylus, the majority of tri-nucleotide repeats ranged from 15 to $24 \mathrm{bp}$ in length (Table 4).

Figure 1 shows the frequency of the identified SSR repeat types. The di-nucleotide repeats (AG/CT)n were predominant, representing $46.35 \%$ of all of the characterized motifs, followed by the (AT/AT)n repeats $(12.93 \%)$ and (AC/GT)n repeats $(4.50 \%)$. For the tri-nucleotide repeats, (AAG/CTT) $\mathrm{n}$ repeats were dominant, representing $8.43 \%$ of all of the characterized motifs, followed by the (AAT/ATT)n repeats (3.93\%), (ATC/ATG)n repeats (2.74\%), (AGG/CCT)n repeats $(2.56 \%)$ and (ACC/GGT)n repeats $(2.45 \%)$. The di-nucleotide repeats (CG/CG)n were the fewest, representing $0.04 \%$ of all of the characterized motifs. These above results indicate that transcriptome sequencing is a useful tool for SSR identification in Corylus.

\section{Development of novel tri-nucleotide SSR markers in Corylus and polymorphism testing}

A total of 160 primer pairs were specially designed from the sequences with tri-nucleotide repeat types, which were tested for amplification in the Ping'ou hybrid hazelnut 'Dawei'. Of these primer pairs, 122 amplified repeatedly and reliably, while the other primer pairs produced too many undesirable bands or no bands and were excluded from further analysis. The percentage of the amplification efficiency of the primers was $76.25 \%$. To validate the level of polymorphism for the newly designed SSR markers and to investigate the transferability, all of the efficient primer pairs were tested on 13 samples of 3 different species of Corylus (Table 2). Electrophoresis on $6 \%$ denatured polyacrylamide gels showed that 22 primer pairs generated perfect polymorphic PCR products among the 13 samples. Characterization data for the 22 polymorphic loci is summarized in Table 5 . The ratio of the polymorphic amplification of the primers was $13.75 \%$. The PIC value ranged from 0.34 for CAF- 22 to 0.62 for CAF- 1 and CAF-2, with the average value of 0.50 . The PIC values for CAF-1 to CAF-11 were higher than 0.50 , which were highly informative. The number of alleles ranged from 3 to 5 per locus (mean 3.75) among the 13 samples. Among these primers, CAF-1, CAF-2, CAF-3, CAF-4, CAF-6, CAF-8, CAF-10, CAF11, CAF-12, CAF-13 and CAF-19 showed transferability in Ping'ou hybrid hazelnuts ( $C$. heterophylla Fisch. $\times$ C. avellana L.), Ping hazelnuts (C. heterophylla Fisch.) and European hazelnuts (C. avellana L.), with a percentage of $55.00 \%$ among the polymorphic primers. CAF-7, CAF-9, CAF-14, CAF-17, CAF-21 and CAF-22 showed transferability in Ping'ou hybrid hazelnuts and Ping hazelnuts. CAF- 5 and CAF-20 showed transferability in Ping'ou hybrid hazelnuts and European hazelnuts. CAF-15 and CAF-18 showed transferability in Ping hazelnuts and European hazelnuts. However, primer CAF-16 showed polymorphic amplification only in Ping'ou hybrid hazelnuts. Figure 2 shows 4 groups of PCR products of polymorphism tri-nucleotide repeat EST-SSRs. The data above indicate that the 22 novel tri-nucleotide EST-SSR markers were reasonably polymorphic in Corylus and might be useful for other genetic studies.

\section{The application of the developed tri-nucleotide repeats SSR markers}

We observed 82 alleles in the 13 samples using 22 developed polymorphic SSR primer pairs. A dendrogram depicting the genetic relations among the 13 samples from three species of Corylus was constructed. They were clustered 


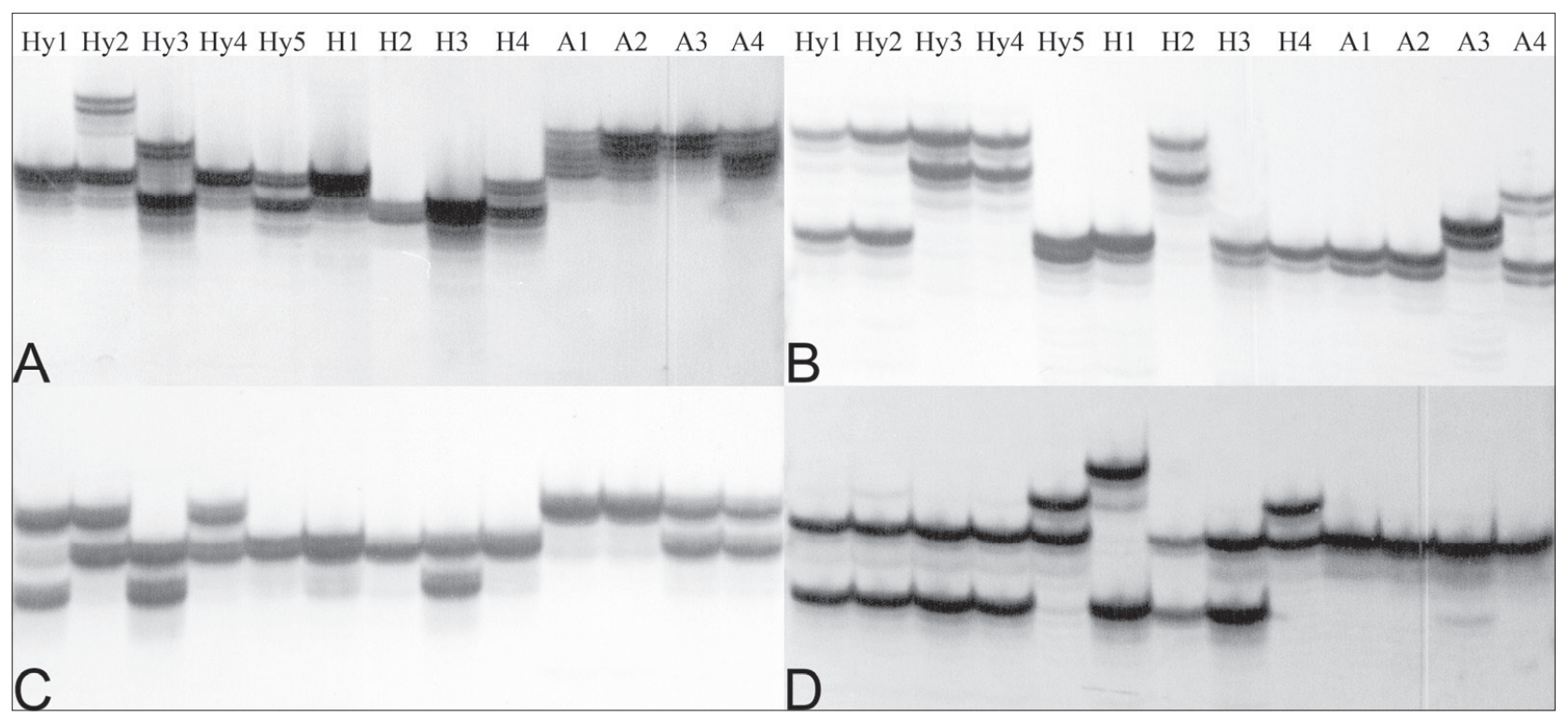

FIGURE 2. PCR products of polymorphism tri-nucleotide repeat EST-SSRs.

into 3 main groups that were in accordance with the species (Figure 3). Group I contained 5 main cultivars of the Ping'ou hybrid hazelnut. It was subdivided into 4 subgroups. 'Dawei' and 'Yuzhui' were included in one subgroup. 'Liaozhen No. 7', 'Liaozhen No. 3' and 'Liaozhen No. 8' were subdivided as single subgroups. Group II contained the 4 varieties of wild Ping hazelnuts, which included 2 subgroups: one comprised 2 varieties, which were collected from Zhalantun, the Inner Mongolia Autonomous Region and Dunhua, Jilin Province, and the other comprised 2 varieties collected from Tieling, Liaoning Province and Weichang, Hebei Province. Finally, group III included 4 cultivars of European hazelnuts, collected from the NCGR in Corvallis, Oregon, USA. It was subdivided into 3 subgroups: the Italian progeny 'Willamette' and 'Tombul' and one of the main cultivars from Turkey were included in one subgroup. 'Tonda Gentile delle Langhe', one of the main cultivars from Italy, and 'Hall's Giant', which originated in Germany, were subdivided as the other two single subgroups.

\section{Discussion}

\section{Frequency and distribution of SSRs in Corylus pistil transcriptome}

Polymorphic SSR markers are important tools for research in genetic diversity, relatedness, evolution, linkage mapping, comparative genomics and gene-based association studies. Deep transcriptome sequencing provides a good resource for the development of SSRs because of the enormous amount of sequence data. Moreover, since the transcriptome is the expressed portion of the genome, markers developed from it should be in or very near the functional regions. Just as the recent reported SSR markers from transcriptome sequences of European hazelnut, 109 polymorphic SSR markers were developed from bark, catkin, leaf and the young seedling transcriptome sequences (Colburn et al., 2017). The SSR markers derived from the pistil transcriptome would increase the likelihood of developing useful markers that are

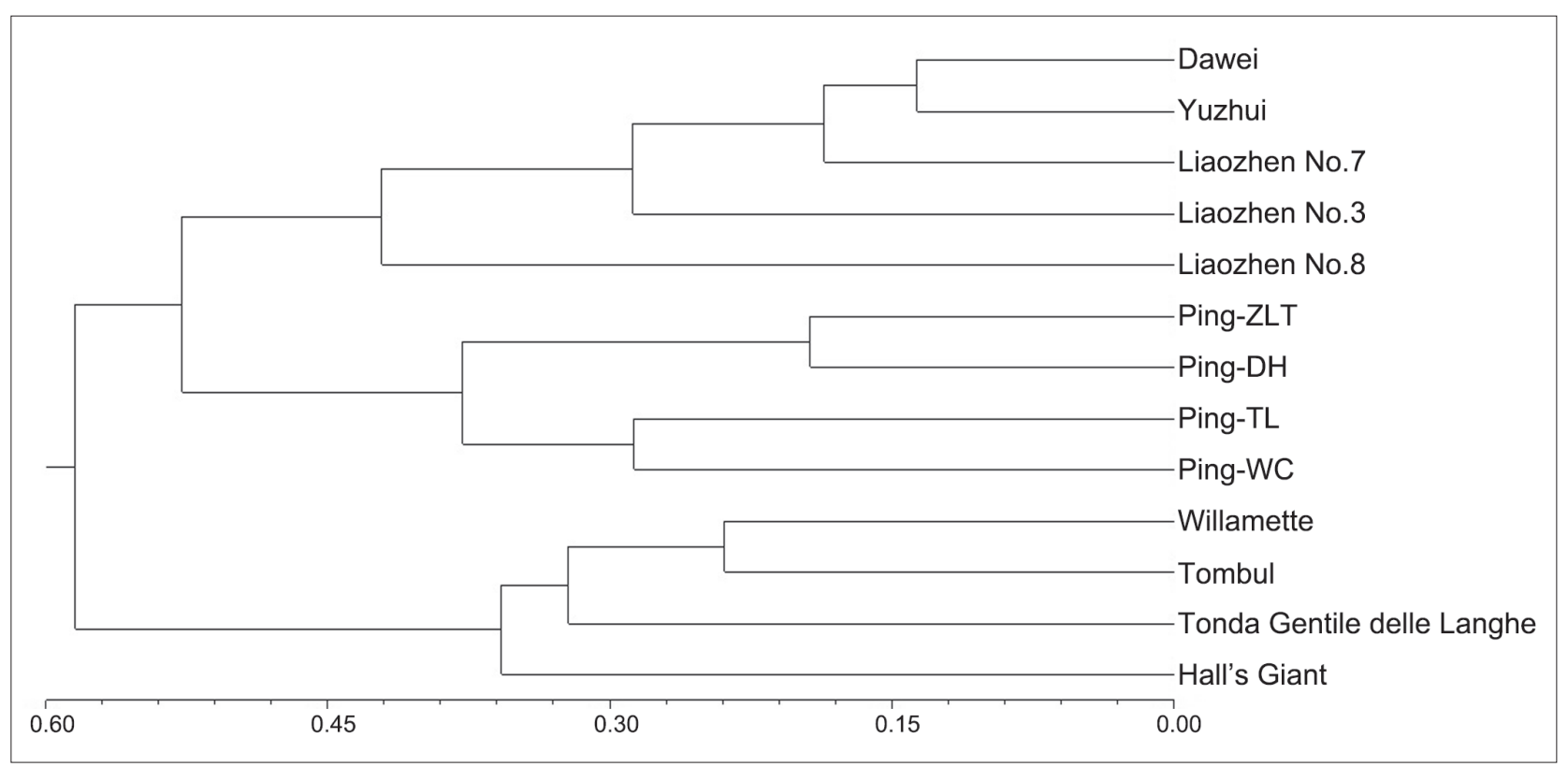

FIGURE 3. UPGMA dendrogram of 13 tested samples based on alleles at 22 developed SSR loci. 
closely linked to functional genes, especially during the pollen-stigma recognition.

In this study, 16,339 sequences were identified containing SSRs from 82,847 unigenes (the total size was 108,232,640), resulting in a percentage of $19.72 \%$. Moreover, the SSR locus was identified for every $5.67 \mathrm{~kb}$ of the examined sequences. The frequency of SSRs from the assembled unigenes and the distribution density of SSRs differ greatly between the taxa in the previous studies. The SSR frequency $(19.72 \%)$ in this study was higher than $14.4 \%$ in species of Amorphophallus (Araceae) (Zheng et al., 2013), 16.67\% in tea (Camellia sinensis) (Tan et al., 2013), 17.21\% (SSRs $\geq 15 \mathrm{bp}$ ) in sesame (Sesamum indicum L.) (Zhang et al., 2012), and slightly lower than $23.79 \%$ in radishes (Raphanus sativus L.) (Wang et al., 2012). For the distribution density, the results of this study were lower than those of the previous study in radishes (one per $3.45 \mathrm{~kb}$ ) (Wang et al., 2012), tea (Camellia sinensis L., one per $3.55 \mathrm{~kb}$ ) (Sharma et al., 2013), species of Amorphophallus (one per $3.63 \mathrm{~kb}$ ) (Zheng et al., 2013), and Chinese jujube (Ziziphus jujuba Mill., one per $3.87 \mathrm{~kb}$ ) (Li et al., 2014). However, the distribution density of SSRs in Corylus was higher than that in the previous study in sesame (one per $6.55 \mathrm{~kb}$ for $15 \mathrm{bp}$ and one per $10.81 \mathrm{~kb}$ for $18 \mathrm{bp}$ ) (Zhang et al., 2012), Chinese medicinal plant (Epimedium sagittatum (Sieb. et Zucc.) Maxim, one per $6.69 \mathrm{~kb}$ ) (Zeng et al., 2010), and Chinese Cymbidium (Cymbidium ensifolium) floral transcriptome (one per $17.56 \mathrm{~kb}$ ) (Li et al., 2013). The frequency of SSRs in various plant genomes was influenced by database-mining tools, the data set size, and the search parameters used to search the SSRs, such as the repeat length threshold and the number of repeat motifs unit (Varshney et al., 2005; Wei et al., 2008). The results showed that the SSR resources in the Corylus transcriptome were at the middle level among many other species, and the number of SSRs was sufficient for identifying EST-SSR markers.

As for the repeat types of the SSRs, di-nucleotide repeat motifs were the most frequent repeat type, representing $63.82 \%$ of the SSR loci identified, followed by tri-nucleotide repeats $(24.54 \%)$, and the higher nucleotide repeat motifs, which were less frequent. This is consistent with the ESTSSRs distributions reported in sesame (Zhang et al., 2012), tea (Tan et al., 2013), species of Amorphophallus (Zheng et al., 2013), and Chinese Cymbidium floral transcriptome (Li et al., 2013). However, tri-nucleotide repeats were predominant in radish species (Shirasawa et al., 2011; Wang et al., 2012). As the similar research work in Betulaceae, the data of di-nucleotide repeats and tri- nucleotide repeats were $63.9 \%$ and $33.8 \%$, respectively, reported by Boccacci et al. (2015) when studying on the Betulaceae EST sequences. While Colburn et al. (2017) reported of the total number of repeats mined from the four hazelnut transcriptomes, $52 \%$ were di-nucleotide repeats, $45 \%$ tri-nucleotide repeats, while the higher repeat motifs process lower frequencies. Trinucleotide repeats are predominant compared with other motifs in coding sequences. This high frequency is likely due to a selective disadvantage of non-trimeric SSR variants in coding regions and the resulting frameshift mutations (Metzgar et al., 2000).

The most abundant di- and tri-nucleotide repeat motifs were AG/CT (46.35\%) and AGG/CCT (8.43\%), respectively, in our transcriptome sequences. The same results were also found in other plant species (Wei et al., 2008; Wang et al., 2012; Zhang et al., 2012; Zheng et al., 2013; Tan et al., 2013). In the research of Boccacci et al. (2015), the most common motif of the di-nucleotide repeats was AG/CT (51.1\%) and the tri-motifs was AAG/CTT (13.2). The most common SSR motifs in the hazelnut transcriptome, Colburn et al. (2017) reported, were AG $(35.8 \%)$ and AAG (12.7\%). The CG/GC SSRs were the least abundant motifs in this study, accounting for $0.04 \%$ of the total SSRs. A low frequency of CG/GC repeats was also observed in the ESTs of other plants (Wei et al., 2011; Wang et al., 2012; Ueno et al., 2012; Tan et al., 2013) and GC/GC repeats were also not found in some species (Kantety et al., 2002; Liang et al., 2009; Zeng et al., 2010; Zhang et al., 2012).

\section{Polymorphism and genera transferability of the developed SSR markers}

In this study, a high success ratio of amplification efficiency $(76.25 \%)$ was obtained compared with similar studies, and this ratio was similar to that observed for trinucleotide repeats in tea (Camellia sinensis L., 72\%, Sharma et al., 2013) and Chinese jujube (71.6\%, Li et al., 2014). All of the novel SSR primers showed good transferability to other cultivars of the Ping'ou hybrid hazelnut $(C$. heterophylla Fisch. $\times C$. avellana L.) and even to the closely related species Ping hazelnut (C. heterophylla Fisch.) and European hazelnut (C. avellana L.). Of these markers, 22 generated perfect polymorphic PCR products, with a percentage of $13.75 \%$. Among these, 20 markers showed polymorphism in the Ping'ou hybrid hazelnut (Hy, in Table 5), 19 markers showed polymorphism in the Ping hazelnut $(\mathrm{H}$, in Table 5) and 15 markers in the European hazelnut (A, in Table 5). Generally, due to the conserved nature of EST-SSR, fewer polymorphisms were detected for EST-SSRs than for the genomic SSRs (Eujayl et al., 2004). However, a high polymorphic rate was also observed in some of the EST-SSR studies (Zheng et al., 2013; Tan et al., 2013; Li et al., 2014). The ratio of marker polymorphisms varies among previous studies. For example, $76.3 \%$ was observed in the Chinese jujube (Li et al., 2014), 47.5\% in Lentils (Lens culinaris Medik.) (Kaur et al., 2011), and 12.9\% was detected in pigeon peas (Cajanus cajan (L.) Millspaugh) (Dutta et al., 2011). The polymorphism ratio mainly depends on the tested samples and the selection criteria. In similar studies in peanut (Arachis hypogaea L.), 26 (10.3\%) EST-SSRs exhibited polymorphisms between 22 cultivated peanut accessions and 221 (88\%) were polymorphic between 16 wild peanut species (Liang et al., 2009); in sesame, 32 (11.59\%) genic-SSR markers were polymorphic in 24 cultivars, 18 $(6.52 \%)$ were polymorphic in one mapping population, and 167 (60.51\%) were polymorphic between the 24 accessions and a wild accession (Zhang et al., 2012). The use of more sensitive techniques for DNA fragment size analysis, e.g., capillary electrophoresis, may be expected to show a higher rate of polymorphism.

In this study, only tri-nucleotide repeats were adopted for developing the SSR markers. Tri-nucleotide repeats and other longer core motifs could be more integrated and could significantly reduce arbitrary binning decisions than di-nucleotide repeats (Cipriani et al., 2008). Capillary electrophoresis showed that the tri-nucleotide repeats were more robust than the di-nucleotide repeats (Amos et al., 2007; Cipriani et al., 2008; Li et al., 2014). Tri-nucleotide core repeats are less frequent in most plant genomes (Zane et al., 2002; Morgante et al., 2002); therefore, the development of tri-nucleotide repeats was more convenient when taking advantage of the high-throughput transcriptome sequencing. Genetic markers are based on particularly expressed sequences; thus, they are potentially tightly linked with functional genes that may control certain important phenotypic characteristics (Ramchiary et al., 2011). The results of many similar stud- 
ies indicate that large numbers of polymorphic SSR markers can be obtained when large volumes of transcript sequences are used. Compared with other SSR detection methods, the de novo RNA sequencing approach was well suited for developing large numbers of genic-SSRs, which could be useful for studying functional diversity, executing marker assisted gene selection and performing association mapping studies in Corylus.

\section{The application of the developed tri-nucleotide repeats SSR markers in three Corylus species}

A total of 82 alleles were detected, using 22 developed polymorphic SSR primer pairs. The PIC value ranged from 0.34 to 0.62 , with an average of 0.50 . Eleven loci were equal to or higher than 0.50 , ranged from 0.50 to 0.62 , which were highly informative. In the similar research on hazelnut, 20 polymorphic SSR loci were amplified in 18 samples, the PIC ranged from 0.14 to 0.81 , and its mean value was 0.50 (Boccacci et al., 2015). The level of polymorphism was slightly higher than in this study. On the contrary, in both the two studies, the SSR primer pairs revealed less polymorphism comparing with gSSRs used in hazelnut germplasm characterization. Boccacci et al. (2005) developed 18 gSSR and showed a high level of polymorphism in 20 accessions, with the PIC value of 0.64. Bassil et al. (2005a) analyzed 25 gSSR loci in 20 genotypes and the PIC were 0.64 . The lower polymorphism of EST-SSRs is usually explained by the location of the genic SSRs in transcribed and hence conserved regions of the genome (Boccacci et al., 2015).

The dendrogram generated from UPGMA cluster analysis was clustered into three main groups related to the species (Figure 3). The hybrids of the Ping'ou hybrid hazelnuts (C. heterophylla Fisch. $\times$ C. avellana L.) were made from 1980 to 1985 by W.J.L. and resulted in a population of more than 2,000 seedlings (Liang et al., 2012). The cultivars were released from 2004 to recent years. The female parents consisted of five advanced selections from wild Ping hazelnuts (C. heterophylla Fisch.) from northeast China. The male parents were the mixed pollen collected from 10 seedlings of the European hazelnuts introduced from Italy. Therefore, the genetic relationship of the Ping'ou hybrid hazelnuts was very complex. It was difficult to detect the parentage relationship of these hybrids, but the mix of the cultivars/varieties is one of the problems in hazelnut production in China. In this study, we developed 20 polymorphic SSR primer pairs for Ping'ou hybrid hazelnuts, and they could successfully distinguish among the five tested cultivars.

The Ping hazelnut is one of the wild species native to China and is widely distributed in the northeast part of China. The propagation pattern of the Ping hazelnut, both by sexual propagation and by clonal propagation, leads to the high genetic diversity of this species. The 4 tested samples used in the present study were collected from Zhalantun $\left(121^{\circ} 53^{\prime} \mathrm{E}\right.$; $\left.48^{\circ} 08^{\prime} \mathrm{N}\right)$, the Inner Mongolia Autonomous Region, Dunhua $\left(128^{\circ} 23^{\prime} \mathrm{E}\right.$; $\left.43^{\circ} 36^{\prime} \mathrm{N}\right)$, Jilin Province, Tieling (124ำ $17^{\prime} \mathrm{E}$; $\left.42^{\circ} 44^{\prime} \mathrm{N}\right)$, Liaoning Province and Weichang $\left(117^{\circ} 23^{\prime} \mathrm{E}\right.$; $42^{\circ} 08^{\prime} \mathrm{N}$ ), and Hebei Province. We developed 19 polymorphic SSR primer pairs for Ping hazelnuts, and the clustering result is in accordance with the geographical distance.

As for the four tested samples of European hazelnuts, 'Willamette' was the Italian progeny (Mehlenbacher et al., 1991), 'Hall's Giant' originated in Germany (Mehlenbacher, 1991), 'Tonda Gentile delle Langhe' was one of the main cultivars from Italy, and 'Tombul' was one of the main cultivars from Turkey. We developed 15 polymorphic SSR primer pairs for $C$. avellana $\mathrm{L}$. The clustering result in the present study was similar to the result reported from another related article (Boccacci et al., 2006). The above data indicate that our 22 SSR markers were reasonably polymorphic in the three species of hazelnuts and might be useful for germplasm characterization and genetic mapping.

\section{Conclusions}

In this study, we used the previously constructed pistil transcriptome to analyze the SSR information and develop novel tri-nucleotide SSR markers in Corylus. We identified 16,339 sequences containing SSRs, resulting in a percentage of $19.72 \%$ in the whole database. SSR locus was identified for every $5.67 \mathrm{~kb}$ of the examined sequences. SSR information, such as the distribution of different nucleotide repeat types, the type, number and percentage of SSRs and the frequency of identified SSR repeat types, were analyzed in detail. Furthermore, 22 genic SSR primer pairs that could generate perfect polymorphic PCR products among the 13 samples were developed, with an average PIC value of 0.50. A dendrogram based on 82 alleles of the 13 samples using the developed SSR markers was generated. The samples were clustered into three main groups in accordance with the species and showed complete separation in the dendrogram.

In summation, the results in this study further highlight the efficient identification of SSRs from the pistil transcriptome of Corylus, indicating that transcriptome sequencing is an effective tool for the development of SSR markers. The developed SSR markers will help in genetic and breeding studies in Corylus and related species.

\section{Acknowledgments}

This work was funded by the National Natural Science Foundation of China (Grant No. 31500560), the Special Fund for Basic Scientific Research Business of Central Public Research Institutes of the Chinese Academy of Forestry (Grant No. CAFYBB2016QB002) and the Basic Research Fund of RIF (Grant No. RIF-12).

\section{Competing interests}

The authors have declared that no competing interests exist.

\section{References}

Amos, W., Hoffman, J.I., Frodsham, A., Zhang, L., Best, S., and Hill, A.V.S. (2007). Automated binning of microsatellite alleles: problems and solutions. Mol. Ecol. Notes 7, 10-14. https://doi.org/10.1111/ j.1471-8286.2006.01560.x.

Bassil, N.V., Botta, R., and Mehlenbacher, S.A. (2005a). Microsatellite markers in Hazelnut: isolation, characterization, and cross-species amplification. J. Am. Soc. Hortic. Sci. 130(4), 543-549. https://doi. org/10.21273/JASHS.130.4.543.

Bassil, N.V., Botta, R., and Mehlenbacher, S.A. (2005b). Additional microsatellite markers of the European hazelnut. Acta Hortic. 686, 105-110. https://doi.org/10.17660/ActaHortic.2005.686.13.

Bassil, N.V., Boccacci, P., Botta, R., Postman, J., and Mehlenbacher, S.A. (2013). Nuclear and chloroplast microsatellite markers to assess genetic diversity and evolution in hazelnut species, hybrids and cultivars. Genet. Resour. Crop Evol. 60, 543-568. https://doi. org/10.1007/s10722-012-9857-z.

Beltramo, C., Boccacci, P., Sandoval, P.M.A., Portis, E., and Botta, R. (2014). Development of a genetic linkage map in hazelnut (Corylus avellana L.) for the detection of QTLs. Acta Hortic. 1052, 99-104. https://doi.org/10.17660/ActaHortic.2014.1052.12. 
Boccacci, P., Akkak, A., Bassil, N.V., Mehlenbacher, S.A., and Botta, R. (2005). Characterization and evaluation of microsatellite loci in European hazelnut (Corylus avellana L.) and their transferability to other Corylus species. Mol. Ecol. Notes 5, 934-937. https://doi. org/10.1111/j.1471-8286.2005.01121.x.

Boccacci, P., Akkak, A., and Botta, R. (2006). DNA-typing and genetic relationships among European hazelnut (Corylus avellana L.) cultivars using microsatellite markers. Genome 49, 598-611. https://doi.org/10.1139/g06-017.

Boccacci, P., Aramini, M., Valentini, N., Bacchetta, L., Rovira, M., Drogoudi, P., Silva, A.P., Solar, A., Calizzano, F., Erdoğan, V., Cristofori, V., Ciarmiello, L.F., Contessa, C., Ferreira, J.J., Marra, F.P., and Botta, R. (2013). Molecular and morphological diversity of on-farm hazelnut (Corylus avellana L.) landraces from southern Europe and their role in the origin and diffusion of cultivated germplasm. Tree Genet. Genomes 9, 1465-1480. https://doi.org/10.1007/s11295-0130651-7.

Boccacci, P., Beltramo, C., Sandoval, P.M.A., Lembo, A., Sartor, C., Mehlenbacher, S.A., Botta, R., and Marinoni, D.T. (2015). In silico mining, characterization and cross-species transferability of EST-SSR markers for European hazelnut (Corylus avellana L.). Mol. Breeding 35, 21. https://doi.org/10.1007/s11032-015-0195-7.

Chen, Z.D., Manchester, S.R., and Sun, H.Y. (1999). Phylogeny and evolution of the Betulaceae as inferred from DNA sequences, morphology, and paleobotany. Am. J. Bot. 86, 1168-1181. https:// doi.org/10.2307/2656981

Cipriani, G., Marrazzo, M.T., Di Gaspero, G., Pfeiffer, A., Morgante, M., and Testolin, R. (2008). A set of microsatellite markers with long core repeat optimized for grape (Vitis spp.) genotyping. BMC Plant Biol. 8, 127. https://doi.org/10.1186/1471-2229-8-127.

Colburn, B.C., Mehlenbacher, S.A., and Sathuvalli, V.R. (2017). Development and mapping of microsatellite markers from transcriptome sequences of European hazelnut (Corylus avellana L.) and use for germplasm characterization. Mol. Breeding 37, 16. https://doi.org/10.1007/s11032-016-0616-2.

Dutta, S., Kumawat, G., Singh, B.P., Gupta, D.K., Singh, S., Dogra, V., Gaikwad, K., Sharma, T.R., Raje, R.S., Bandhopadhya, T.K., Datta, S., Singh, M.N., Bashasab, F., Kulwal, P., Wanjari, K.B., Varshney, R.K., Cook, D.R., and Singh, N.K. (2011). Development of genic-SSR markers by deep transcriptome sequencing in pigeonpea [Cajanus cajan (L.) Millspaugh]. BMC Plant Biol. 11, 17. https://doi.org/10.1186/14712229-11-17.

Eujayl, I., Sledge, M.K., Wang, L., May, G.D., Chekhovskiy, K., Zwonitzer J.C., and Mian, M.A.R. (2004). Medicago truncatula EST-SSRs reveal cross-species genetic markers for Medicago spp. Theor. Appl. Genet. 108, 414-422. https://doi.org/10.1007/s00122-003-1450-6.

Gürcan, K., and Mehlenbacher, S.A. (2010a). Transferability of microsatellite markers in the Betulaceae. J. Am. Soc. Hortic. Sci. 135, 159-173. https://doi.org/10.21273/JASHS.135.2.159.

Gürcan, K., and Mehlenbacher, S.A. (2010b). Development of microsatellite marker loci for European hazelnut (Corylus avellana L.) from ISSR fragments. Mol. Breeding 26, 551-559. https://doi. org/10.1007/s11032-010-9464-7.

Gürcan, K., Mehlenbacher, S.A., Botta, R., and Boccacci, P. (2010a). Development, characterization, segregation, and mapping of microsatellite markers for European hazelnut (Corylus avellana L.) from enriched genomic libraries and usefulness in genetic diversity studies. Tree Genet. Genomes 6, 513-531. https://doi.org/10.1007/ s11295-010-0269-y.

Gűrcan, K., Mehlenbacher, S.A., and Erdoğan, V. (2010b). Genetic diversity in hazelnut (Corylus avellana L.) cultivars from Black Sea countries assessed using SSR markers. Plant Breeding 129, 422-434. https://doi.org/10.1111/j.1439-0523.2009.01753.x.

Kantety, R.V., Rota, M.L., and Matthews, D.E. (2002). Data mining for simple sequence repeats in expressed sequence tags from barley, maize, rice, sorghum and wheat. Plant Mol. Biol. 48, 501-510. https://doi.org/10.1023/A:1014875206165.

Kaur, S., Cogan, N.O., Pembleton, L.W., Shinozuka, M., Savin, K.W., Materne, M., and Forster, J.W. (2011). Transcriptome sequencing of lentil based on second-generation technology permits large-scale unigene assembly and SSR marker discovery. BMC Genomics 12, 265. https://doi.org/10.1186/1471-2164-12-265.

Li, X., Luo, J., Yan, T., Xiang, L., Jin, F., Qin., D., Sun, C., and Xie, M. (2013). Deep sequencing-based analysis of the Cymbidium ensifolium floral transcriptome. PLoS ONE 8(12), e85480. https://doi.org/10.1371/ journal.pone.0085480.

Li, Y.Y., Xu, C.Q., Lin, X.G., Cui, B.B., Wu, R.L., and Pang, X.M. (2014) De novo assembly and characterization of the fruit transcriptome of Chinese jujube (Ziziphus jujuba Mill.) using 454 pyrosequencing and the development of novel tri-nucleotide SSR markers. PLoS ONE 9(9), e106438. https://doi.org/10.1371/journal.pone.0106438.

Liang, W.J., Dong, D.F., Wang, G.X., Dong, F.X., Liang, L.S., and Ma, Q.H. (2012). Progresses on the hazelnut cross breeding of Corylus heterophylla Fisch. $\times$ Corylus avellana L. in China. Acta Hortic. 940, 233-238. https://doi.org/10.17660/ActaHortic.2012.940.31.

Liang, X.Q., Chen, X.P., Hong, Y.B., Liu, H.Y., Zhou, G.Y., Li, S.X., and Guo, B.Z. (2009). Utility of EST derived SSR in cultivated peanut (Arachis hypogaea L.) and Arachis wild species. BMC Plant Biol. 9, 35. https:// doi.org/10.1186/1471-2229-9-35

Luro, F.L., Costantino, G., Terol, J., Argout, X., Allario, T., Wincker, P., Talon, M., Ollitrault, P., and Morillon, R. (2008). Transferability of the EST-SSRs developed on Nules clementine (Citrus clementina Hort ex Tan) to other Citrus species and their effectiveness for genetic mapping. BMC Genomics 9(1), 287. https://doi.org/10.1186/14712164-9-287.

Ma, Q.H., Huo, H.L., Chen, X., Zhao, T.T., Liang, W.J., and Wang, G.X. (2014). Study on the taxonomy, distribution, development and utilization of Corylus kweichowensis Hu. J. Plant Genet. Res. 15(6), 1223-1231 (in Chinese with English abstract).

Mardis, E.R. (2008). The impact of next-generation sequencing technology on genetics. Trends Genet. 24(3), 133-141. https://doi. org/10.1016/j.tig.2007.12.007.

Mehlenbacher, S.A. (1991). Hazelnuts (Corylus). Genetic resources of temperate fruit and nut crops. Acta Hortic. 290, 791-836. https:// doi.org/10.17660/ActaHortic.1991.290.18.

Mehlenbacher, S.A. (2003). Hazelnuts. In A Guide to Nut Tree Culture in North America, Vol. 1, D.W. Fulbright, ed. (Northern Nut Growers Association), p. 183-215.

Mehlenbacher, S.A., Miller, A.N., Thompson, M.M., Lagerstedt, H.B., and Smith, D.C. (1991). 'Willamette' hazelnut. HortScience 26, 13411342. https://doi.org/10.21273/HORTSCI.26.10.1341.

Mehlenbacher, S.A., Brown, R.N., Nouhra, E.N., Gökirmak, T., Bassil, N.V., and Kubisiak, T.L. (2006). A genetic linkage map for hazelnut (Corylus avellana L.) based on RAPD and SSR markers. Genome 49, 122-133. https://doi.org/10.1139/g05-091.

Metzgar, D., Bytof, J., and Wills, C. (2000). Selection against frameshift mutations limits microsatellite expansion in coding DNA. Genome Res. 10, 72-80.

Metzker, M.L. (2010). Sequencing technologies-the next generation. Nat. Rev. Genet. 11(1), 31-46. https://doi.org/10.1038/nrg2626.

Morgante, M., Hanafey, M., and Powell, W. (2002). Microsatellites are preferentially associated with nonrepetitive DNA in plant genomes. Nat. Genet. 30, 194-200. https://doi.org/10.1038/ng822.

Morozova, O., and Marra, M.A. (2008). Applications of next-generation sequencing technologies in functional genomics. Genomics 92(5), 255-264. https://doi.org/10.1016/j.ygeno.2008.07.001. 
Muehlbauer, M.F., Honig, J.A., Capik, J.M., Vaiciunas, J.N., and Molnar, T.J. (2014). Characterization of eastern filbert blight resistant Hazelnut germplasm using microsatellite markers. J. Am. Soc. Hortic. Sci. 139(4), 399-432. https://doi.org/10.21273/JASHS.139.4.399.

Pang, X.M., Hu, C.G., and Deng, X.X. (2003). Phylogenetic relationships among citrus and its relatives as revealed by SSR markers. Acta Genetica Sinica. 30(1), 81-87.

Poncet, V., Rondeau, M., Tranchant, C., Cayrel, A., Hamon, S., de Kochko, A., and Hamon, P. (2006). SSR mining in coffee tree EST databases: potential use of EST-SSRs as markers for the Coffea genus. Mol. Genet. Genomics 276(5), 436-449. https://doi.org/10.1007/ s00438-006-0153-5.

Powell, W., Machray, G.C., and Provan, J. (1996). Polymorphism revealed by simple sequence repeats. Trends Plant Sci. 1, 215-222. https://doi.org/10.1016/S1360-1385(96)86898-0.

Ramchiary, N., Nguyen, V.D., Li, X.N., Hong, C.P., Dhandapani, V., Choi, S.R., Yu, G., Piao, Z.Y., and Lim, Y.P. (2011). Genic microsatellite markers in Brassica rapa: development, characterization, mapping and their utility in other cultivated and wild Brassica relatives. DNA Res. 18, 305-320. https://doi.org/10.1093/dnares/dsr017.

Sathuvalli, V.R., Chen, H., Mehlenbacher, S.A., and Smith, D.C. (2011). DNA markers linked to eastern filbert blight resistance in 'Ratoli' hazelnut (Corylus avellana L.). Tree Genet. Genomes 7, 337-345. https://doi.org/10.1007/s11295-010-0335-5.

Sharma, R.K., Bhardwaj, P., Negi, R., Mohapatra, T., and Ahuja, P.S. (2009). Identification, characterization and utilization of unigene derived microsatellite markers in tea (Camellia sinensis L.). BMC Plant Biol. 9, 53. https://doi.org/10.1186/1471-2229-9-53.

Shi, C.Y., Yang, H., Wei, C.L., Yu, O., Zhang, Z.Z., Jiang, C.J., Sun, J., Li, Y.Y., Chen, Q., Xia, T., and Wan, X.C. (2011). Deep sequencing of the Camellia sinensis transcriptome revealed candidate genes for major metabolic pathways of tea-specific compounds. BMC Genomics 12(1), 131. https://doi.org/10.1186/1471-2164-12-131.

Shirasawa, K., Oyama, M., Hirakawa, H., Sato, S., Tabata, S., Fujioka, T., Kimizuka-Takagi, C., Sasamoto, S., Watanabe, A., Kato, M., Kishida, Y., Kohara, M., Takahashi, C., Tsuruoka, H., Wada, T., Sakai, T., and Isobe, S. (2011). An EST-SSR linkage map of Raphanus sativus and comparative genomics of the Brassicaceae. DNA Res. 18, 221-232. https://doi.org/10.1093/dnares/dsr013.

Smith, J.S.C., Chin, E.C.L., Shu, H., Smith, O.S., Wall, S.J., Senior, M.L., Mitchell, S.E., Kresovich, S., and Ziegle, J. (1997). An evaluation of the utility of SSR loci as molecular markers in maize (Zea mays L.): Comparisons with data from RFLPs and pedigree. Theor. Appl. Genet. 95, 163-173. https://doi.org/10.1007/s001220050544.

Tan, L.Q., Wang, L.Y., Wei, K., Zhang, C.C., Wu, L.Y., Qi, G.N., Cheng, H., Zhang, Q., Cui, Q.M., and Liang, J.B. (2013). Floral transcriptome sequencing for SSR marker development and linkage map construction in the tea plant (Camellia sinensis). PLoS ONE 8(11), e81611. https://doi.org/10.1371/journal.pone.0081611.

Ueno, S., Moriguchi, Y., Uchiyama, K., Ujino-Ihara, T., Futamura, N., Sakurai, T., Shinohara, K., and Tsumura, Y. (2012). A second generation framework for the analysis of microsatellites in expressed sequence tags and the development of EST-SSR markers for a conifer, Cryptomeria japonica. BMC Genomics 13, 136. https:// doi.org/10.1186/1471-2164-13-136.

Varshney, R.K., Graner, A., and Sorrells, M.E. (2005). Genic microsatellite markers in plants: features and applications. Trends Biotechnol. 23(1), 48-55. https://doi.org/10.1016/j. tibtech.2004.11.005.

Wang, J.Y., Pan, L.J., Yang, Q.L., and Yu, S.L. (2010). Development and characterization of EST-SSR markers from NCBI and cDNA library in cultivated peanut (Arachis hypogaea L.). Legume Genomics and Genetics 1, 30-33.
Wang, S.F., Wang, X.F., He, Q.W., Liu, X.X., Xu, W.L., Li, L.B., Gao, J.W., and Wang, F.D. (2012). Transcriptome analysis of the roots at early and late seedling stages using Illumina paired-end sequencing and development of EST-SSR markers in radish. Plant Cell Rep. 31, 14371447. https://doi.org/10.1007/s00299-012-1259-3.

Wang, X.W., Luan, J.B., Li, J.M., Bao, Y.Y., Zhang, C.X., and Liu, S.S. (2010). De novo characterization of a whitefly transcriptome and analysis of its gene expression during development. BMC Genomics 11(1), 400. https://doi.org/10.1186/1471-2164-11-400.

Wei, L.B., Zhang, H.Y., Zheng, Y.Z., Guo, W.Z., and Zhang, T.Z. (2008). Developing EST-derived microsatellites in sesame (Sesamum indicum L.). Acta Agron. Sinica 34(12), 2077-2084. https://doi.org/10.1016/ S1875-2780(09)60019-5.

Wei, W.L., Qi, X.Q., Wang, L.H., Zhang, Y.X., Hua, W., Li, D.H., Lv, H.X., and Zhang, X.R. (2011). Characterization of the sesame (Sesamum indicum L.) global transcriptome using Illumina paired-end sequencing and development of EST-SSR markers. BMC Genomics 12(1), 451. https://doi.org/10.1186/1471-2164-12-451.

Yoo, K.O., and Wen, J. (2002). Phylogeny and biogeography of Carpinus and subfamily Coryloideae (Betulaceae). Int. J. Plant Sci. 163, 641-650. https://doi.org/10.1086/340446.

Zane, L., Bargelloni, L., and Patarnello, T. (2002). Strategies for microsatellite isolation: A review. Mol. Ecol. 11, 1-16. https://doi. org/10.1046/j.0962-1083.2001.01418.x.

Zeng, S.H., Xiao, G., Guo, J., Fei, Z.J., Xu, Y.Q., Roe, B.A., and Wang, Y (2010). Development of a EST dataset and characterization of EST-SSRs in a traditional Chinese medicinal plant, Epimedium sagittatum (Sieb. et Zucc.) Maxim. BMC Genomics 11, 94. https:// doi.org/10.1186/1471-2164-11-94.

Zhang, H.Y., Wei, L.B., Miao, H.M., Zhang, T.D., and Wang, C.Y. (2012). Development and validation of genic-SSR markers in sesame by RNA-seq. BMC Genomics 13, 316. https://doi.org/10.1186/14712164-13-316.

Zheng, X.F., Pan, C., Diao, Y., You, Y.N., Yang, C.Z., and Hu, Z.L. (2013). Development of microsatellite markers by transcriptome sequencing in two species of Amorphophallus (Araceae). BMC Genomics 14, 490. https://doi.org/10.1186/1471-2164-14-490.

Zong, J.W., Zhao, T.T., Ma, Q.H., Liang, L.S., and Wang, G.X. (2015) Assessment of genetic diversity and population genetic structure of Corylus mandshurica in China using SSR markers. PLoS ONE 10(9), e0137528. https://doi.org/10.1371/journal.pone.0137528.

Received: Jun. 8, 2018

Accepted: Nov. 28, 2018

Address of authors:

Tiantian Zhao*, Jingjing Li*, Dingding Miao, Lisong Liang, Guixi Wang and Qinghua Ma**

Research Institute of Forestry, Chinese Academy of Forestry, Key Laboratory of Tree Breeding and Cultivation of the State Forestry and Grassland Administration, Hazelnut Engineering and Technical Research Center of the State Forestry and Grassland Administration, Beijing, 100091, China

* These authors have contributed equally to this work

** Corresponding author; E-mail: mqhmary@sina.com Tel.: +8610 62888537; Fax: +861062872015 


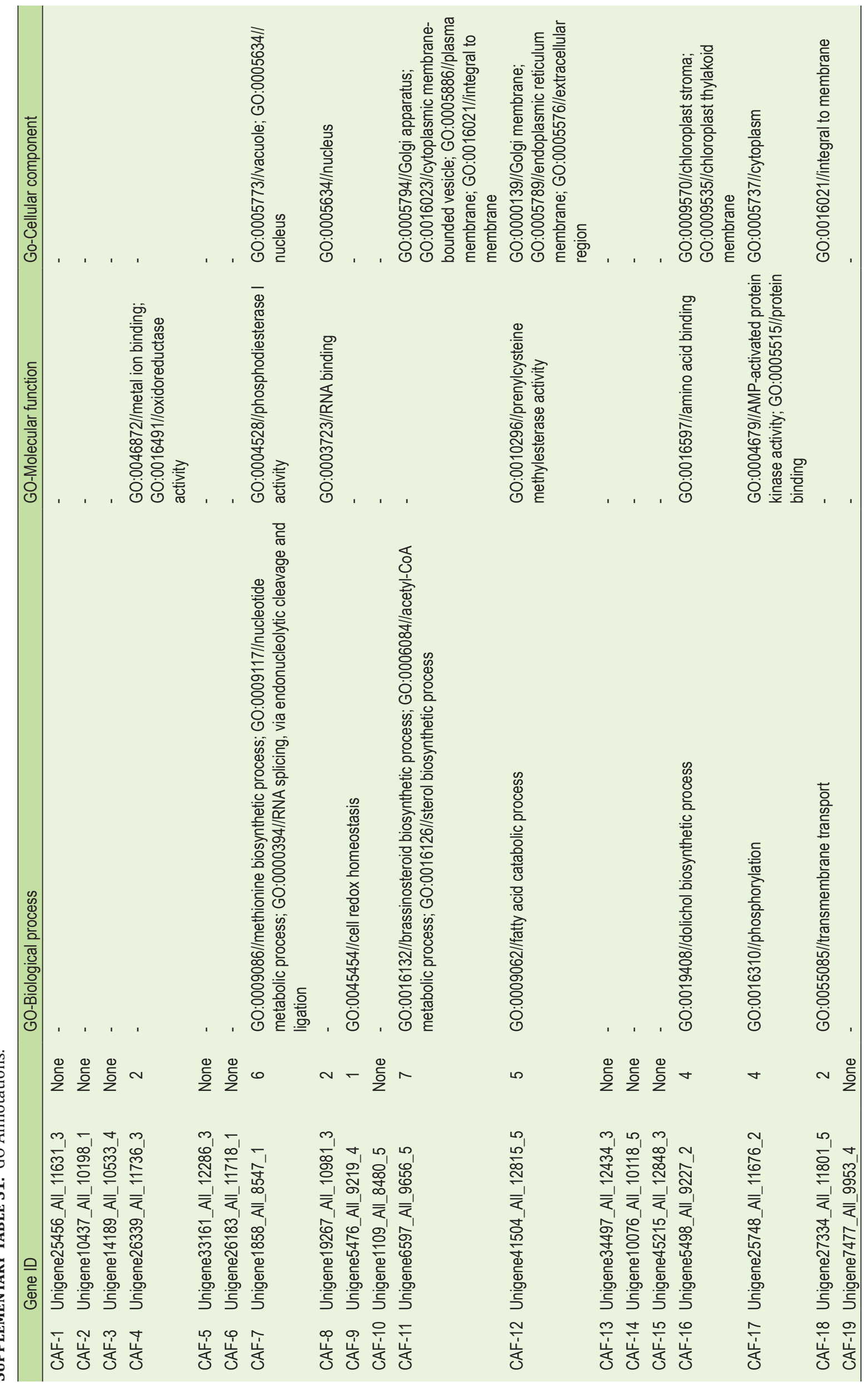




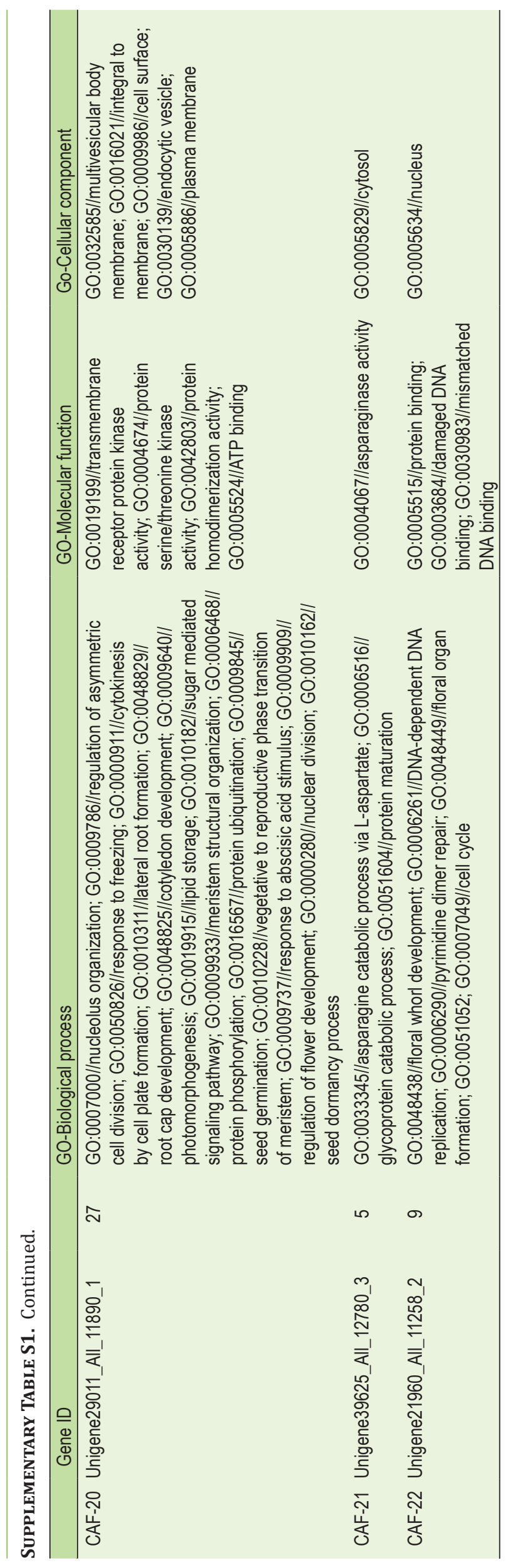

